\title{
On Fractional Model Reference Adaptive Control
}

\author{
Bao Shi, Jian Yuan, and Chao Dong \\ Institute of System Science and Mathematics, Naval Aeronautical and Astronautical University, Yantai 264001, China \\ Correspondence should be addressed to Jian Yuan; yuanjianscar@gmail.com
}

Received 19 August 2013; Accepted 30 October 2013; Published 16 January 2014

Academic Editors: A. Atangana, S. C. O. Noutchie, and A. Secer

Copyright (C) 2014 Bao Shi et al. This is an open access article distributed under the Creative Commons Attribution License, which permits unrestricted use, distribution, and reproduction in any medium, provided the original work is properly cited.

\begin{abstract}
This paper extends the conventional Model Reference Adaptive Control systems to fractional ones based on the theory of fractional calculus. A control law and an incommensurate fractional adaptation law are designed for the fractional plant and the fractional reference model. The stability and tracking convergence are analyzed using the frequency distributed fractional integrator model and Lyapunov theory. Moreover, numerical simulations of both linear and nonlinear systems are performed to exhibit the viability and effectiveness of the proposed methodology.
\end{abstract}

\section{Introduction}

Fractional calculus dates back to the end of the 17th century. Over three hundred years, a firm theoretical foundation has been established due primarily to Liouville, Grünwald, Letnikov, Riemann, and Caputo. In the last three decades, many scientific studies have shown the importance of fractional calculus and its applications in mathematics, physics, chemistry, material, engineering, finance, and even social science [1-3]. The stability of fractional differential equations and fractional control has gained rapid development very recently [4-6].

Several pioneering attempts to develop fractional order control methodologies have been made, such as TID control [7], CRONE control [8], fractional PID control [9], and fractional lead-lag compensator [10]. Basic ideas and technical formulations of the above four fractional control schemes with comparative comments have been addressed in [11]. By applying fractional calculus to advanced nonlinear control theory, several fractional nonlinear control schemes have been proposed very recently, such as fractional sliding mode control [12-24], fractional adaptive control [25-29], and fractional optimal control [30-32]. Particularly, in [25], the authors have presented two ideas to extend the conventional Model Reference Adaptive Control (MRAC) by using fractional order parameter adjustment rule and fractional reference model. In [26], a fractional model reference adaptive control algorithm for SISO plants has been proposed in frequency domain, which guarantees the stability and ability to reject disturbances.

Inspired by contributions from $[25,26]$, this paper aims at going further by applying an incommensurate fractional adaptation law to fractional plant and fractional reference model. Furthermore, the stability and tracking convergence of the fractional adaptive system are analyzed based on the continuous frequency distributed model of fractional integrator.

The rest of the paper is organized as follows. Section 2 reviews some basic definitions and theorems about fractional calculus. Section 3 designs a control law and a fractional adaptation law for fractional linear MRAC systems along with numerical simulations. Section 4 extends the proposed schemes to fractional nonlinear systems. Finally, Section 5 concludes this paper with some remarks on future study.

\section{Basic Definitions and Preliminaries}

Fractional calculus is a generalization of integration and differentiation to noninteger order fundamental operator ${ }_{a} D_{t}^{\alpha}$, where $a$ and $t$ are the bounds of the operation and $a \in \mathbb{R}$. The three most frequently used definitions for the general fractional calculus are the Grünwald-Letnikov definition, 
the Riemann-Liouville definition, and the Caputo definition [1-3].

Definition 1. The Grünwald-Letnikov derivative definition of order $\alpha$ is described as

$$
{ }_{a} D_{t}^{\alpha} f(t)=\lim _{h \rightarrow 0} \frac{1}{h^{\alpha}} \sum_{j=0}^{\infty}(-1)^{j}\left(\begin{array}{c}
\alpha \\
j
\end{array}\right) f(t-j h) .
$$

Definition 2. The Riemann-Liouville derivative definition of order $\alpha$ is described as

$$
{ }_{a} D_{t}^{\alpha} f(t)=\frac{1}{\Gamma(n-\alpha)} \frac{d^{n}}{d t^{n}} \int_{a}^{t} \frac{f(\tau) d \tau}{(t-\tau)^{\alpha-n+1}} .
$$

Definition 3. The Caputo definition of fractional derivatives can be written as

$$
{ }_{a} D_{t}^{\alpha} f(t)=\frac{1}{\Gamma(n-\alpha)} \int_{a}^{t} \frac{f^{(n)}(\tau) d \tau}{(t-\tau)^{\alpha-n+1}}, \quad n-1<\alpha<n .
$$

In the following, we use the Caputo approach to describe fractional systems and the Grünwald-Letnikov approach to perform numerical simulations. To simplify the notation, we denote the fractional derivative of order $\alpha$ as $D^{\alpha}$ instead of ${ }_{0} D_{t}^{\alpha}$ in this paper.

Lemma 4 (the continuous frequency distributed model [33]). The fractional integrator $D^{-\alpha}, 0<\alpha<1$ is a linear frequency distributed system, with input $v(t)$ and output $x(t)$. Its frequency distributed state $z(\omega, t)$ verifies the differential equation (for the elementary frequency $\omega$ ) as follows:

$$
\frac{\partial z(\omega, t)}{\partial t}=-\omega z(\omega, t)+v(t)
$$

and the output $x(t)$ of the fractional integrator is the weighted integral (with weight $\mu(\omega)$ ) of all the contributions $z(\omega, t)$ ranging from 0 to $\infty$ as follows:

$$
x(t)=\int_{0}^{\infty} \mu(\omega) z(\omega, t) d \omega
$$

with $\mu(\omega)=(\sin (\alpha \pi) / \pi) \omega^{-\alpha}$.

The relations (4) and (5) define the frequency distributed model of the fractional integrator.

Lemma 5. The quadratic form $W=W_{1}+W_{2}$ is positive semidefinite if $a_{i} \geq 0$, where $W_{1}=\sum_{i=1}^{m} W_{1 i}$ and $W_{2}=$ $\sum_{i=1}^{m} a_{i} W_{2 i}$ with $W_{1 i}=\int_{0}^{\infty} \mu_{i}(\omega) \omega z_{i}^{2} d \omega$ and $W_{2 i}=x_{i}^{2}, i=1,2$, $\ldots, m[34]$.

\section{Adaptive Control of Fractional Linear Systems}

In this section, we extend the conventional MRAC systems to fractional ones based on the theory of fractional calculus. Firstly, a fractional plant and an incommensurate fractional reference model are described by the fractional differential equations. Then, a control law and an incommensurate fractional adaptation law which are generalized from the conventional ones $[35,36]$ are designed. Finally, the stability and tracking performance of the fractional adaptive system are analyzed based on the continuous frequency distributed model of fractional integrator.

3.1. Fractional Adaptive Control Design. Consider the following fractional differential equation:

$$
D^{\alpha_{1}} y=-a_{p} y+b_{p} u,
$$

where $\alpha_{1}$ is the fractional order lying between $(0,1), y$ is the plant output, $u$ is the input, and $a_{p}$ and $b_{p}$ are constant plant parameters that are assumed to be unknown.

The reference model is specified by a fractional differential equation as follows:

$$
D^{\alpha_{1}} y_{m}=-a_{m} y_{m}+b_{m} r(t)
$$

where $a_{m}$ and $b_{m}$ are constant parameters and $r(t)$ is a bounded external reference signal.

Our objective of the fractional adaptive control design is to construct a control law and a fractional adaptation law to make the fractional plant (6) track the fractional reference model (7) on the basis of system stability.

Let us assume the sign of the parameter $b_{p}$ to be known and design the control law to be

$$
u=\widehat{a}_{r}(t) r+\widehat{a}_{y}(t) y,
$$

where $\widehat{a}_{r}(t)$ and $\widehat{a}_{y}(t)$ are variable feedback gains to be decided later.

Define the tracking error

$$
e=y-y_{m}
$$

and the parameter errors

$$
\begin{aligned}
& \tilde{a}_{r}=\widehat{a}_{r}-a_{r}^{*}, \\
& \tilde{a}_{y}=\widehat{a}_{y}-a_{y}^{*},
\end{aligned}
$$

where $a_{r}^{*}=b_{m} / b_{p}$ and $a_{y}^{*}=\left(a_{p}-a_{m}\right) / b_{p}$ are the same as the conventional case.

Subtracting (7) from (6) derives the dynamics of tracking error as follows:

$$
D^{\alpha_{1}} e=-a_{m} e+b_{p}\left(\tilde{a}_{r} r+\tilde{a}_{y} y\right) .
$$

To adjust the two parameters in the control law (8), an adaptation law can be chosen in the fractional form as follows

$$
\begin{aligned}
& D^{\alpha_{2}} \widehat{a}_{r}=-\operatorname{sign}\left(b_{p}\right) \text { yer }, \\
& D^{\alpha_{3}} \widehat{a}_{y}=-\operatorname{sign}\left(b_{p}\right) \text { yey },
\end{aligned}
$$

where $0<\alpha_{2}<1,0<\alpha_{3}<1$.

Note that the control law (8) and the fractional adaptation law (12) are generalized from the conventional MRAC systems $[35,36]$. 
3.2. Analysis of Stability and Tracking Convergence. In the following, we will prove that the fractional plant (6) can be controlled with the control law (8) and the adaptation law (12).

The tracking error system (11) and adaptation law (12) constitute the following closed-loop adaptive system:

$$
\begin{aligned}
& D^{\alpha_{1}} e=-a_{m} e+b_{p}\left(\tilde{a}_{r} r+\tilde{a}_{y} y\right), \\
& D^{\alpha_{2}} \widehat{a}_{r}=-\operatorname{sign}\left(b_{p}\right) \text { yer, } \\
& D^{\alpha_{3}} \widehat{a}_{y}=-\operatorname{sign}\left(b_{p}\right) \text { yey. }
\end{aligned}
$$

Based on the continuous frequency distributed model of the fractional integrator in Lemma 4, the above adaptive system is exactly equivalent to the infinite dimensional ODEs as follows:

$$
\begin{aligned}
& \frac{\partial z_{1}(\omega, t)}{\partial t}=-\omega z_{1}(\omega, t)-a_{m} e+b_{p}\left(\widetilde{a}_{r} r+\widetilde{a}_{y} y\right), \\
& e(t)=\int_{0}^{\infty} \mu_{1}(\omega) z_{1}(\omega, t) d \omega \\
& \frac{\partial z_{2}(\omega, t)}{\partial t}=-\omega z_{2}(\omega, t)--\operatorname{sign}\left(b_{r}\right) \text { jer }, \\
& \tilde{a}_{r}(t)=\int_{0}^{\infty} \mu_{2}(\omega) z_{2}(\omega, t) d \omega, \\
& \frac{\partial z_{3}(\omega, t)}{\partial t}=-\omega z_{3}(\omega, t)--\operatorname{sign}\left(b_{y}\right) \gamma e y, \\
& \tilde{a}_{y}(t)=\int_{0}^{\infty} \mu_{3}(\omega) z_{3}(\omega, t) d \omega,
\end{aligned}
$$

with $\mu_{i}(\omega)=\left(\sin \left(\alpha_{i} \pi\right) / \pi\right) \omega^{-\alpha_{i}}, i=1,2,3$.

In the above continuous frequency distributed model, $z_{1}(\omega, t), z_{2}(\omega, t)$, and $z_{3}(\omega, t)$ are the true state variables, while $e(t), \tilde{a}_{r}(t)$, and $\widetilde{a}_{y}(t)$ are the pseudo state variables.

Let us define two types of Lyapunov functions as follows:

(i) $v_{i}(w, t)$ : the monochromatic Lyapunov functions corresponding to the elementary frequency;

(ii) $V_{i}(t)$ : the Lyapunov functions summing all the monochromatic $v_{i}(w, t)$ with the weighting functions $\mu_{i}(\omega)$, $i=1,2,3$.

Namely,

$$
\begin{gathered}
v_{1}(w, t)=\frac{1}{2} z_{1}^{2}, \\
v_{2}(w, t)=\frac{\left|b_{p}\right|}{2 \gamma} z_{2}^{2}, \\
v_{3}(w, t)=\frac{\left|b_{p}\right|}{2 \gamma} z_{3}^{2}, \\
V_{i}(t)=\int_{0}^{\infty} \mu_{i}(\omega) v_{i}(\omega, t) d \omega, \quad i=1,2,3 .
\end{gathered}
$$

Then,

$$
\frac{d V_{1}}{d t}=\int_{0}^{\infty} \mu_{1}(\omega) \frac{\partial v_{1}(\omega, t)}{\partial t} d \omega
$$

Substituting the first equation of (14) into (16) gives

$$
\begin{aligned}
& \frac{d V_{1}}{d t}=\int_{0}^{\infty} \mu_{1}(\omega) z_{1}\left[-\omega z_{1}(\omega, t)\right. \\
& \left.\quad-a_{m} e+b_{p}\left(\tilde{a}_{r} r+\tilde{a}_{y} y\right)\right] d \omega \\
& =-\int_{0}^{\infty} \mu_{1}(\omega) \omega z_{1}^{2} d \omega \\
& \quad+\int_{0}^{\infty} \mu_{1}(\omega) z_{1}\left[-a_{m} e+b_{p}\left(\tilde{a}_{r} r+\tilde{a}_{y} y\right)\right] d \omega \\
& =-\int_{0}^{\infty} \mu_{1}(\omega) \omega z_{1}^{2} d \omega+\left[-a_{m} e+b_{p}\left(\tilde{a}_{r} r+\tilde{a}_{y} y\right)\right] \\
& \quad \times \int_{0}^{\infty} \mu_{1}(\omega) z_{1} d \omega .
\end{aligned}
$$

Substituting the second equation of (14) into the integral term of (17) yields

$$
\frac{d V_{1}}{d t}=-\int_{0}^{\infty} \mu_{1}(\omega) \omega z_{1}^{2} d \omega-a_{m} e^{2}+e b_{p}\left(\tilde{a}_{r} r+\tilde{a}_{y} y\right) .
$$

Similarly, one derives

$$
\begin{aligned}
& \frac{d V_{2}}{d t}=-\frac{\left|b_{p}\right|}{\gamma} \int_{0}^{\infty} \mu_{2}(\omega) \omega z_{2}^{2} d \omega-e b_{p} \widetilde{a}_{r} r, \\
& \frac{d V_{3}}{d t}=-\frac{\left|b_{p}\right|}{\gamma} \int_{0}^{\infty} \mu_{3}(\omega) \omega z_{3}^{2} d \omega-e b_{p} \widetilde{a}_{y} y .
\end{aligned}
$$

Finally, lets define

$$
V(t)=V_{1}(t)+V_{2}(t)+V_{3}(t) .
$$

Then, one derives

$$
\begin{aligned}
\frac{d V}{d t}= & -\int_{0}^{\infty} \mu_{1}(\omega) \omega z_{1}^{2} d \omega-\frac{\left|b_{p}\right|}{\gamma} \int_{0}^{\infty} \mu_{2}(\omega) \omega z_{2}^{2} d \omega \\
& -\frac{\left|b_{p}\right|}{\gamma} \int_{0}^{\infty} \mu_{3}(\omega) \omega z_{3}^{2} d \omega-a_{m} e^{2} .
\end{aligned}
$$

Owing to Lemma 5, $d V / d t$ is negative semidefinite, implying the stability of the fractional adaptive system (13).

This proves that the fractional MRAC problem (6)-(7) can be solved by using the control law (8) and the fractional adaptation law (12).

3.3. Numerical Simulations. Consider the control of the fractional plant with known fractional order $\alpha_{1}=0.9$ and unknown parameters $a_{p}$ and $b_{p}$. The sign of $b_{p}$ is assumed to be positive. The fractional reference model is chosen to be

$$
D^{0.9} y_{m}=-4 y_{m}+4 \sin (3 t),
$$

that is, $\alpha_{1}=0.9, a_{m}=4, b_{m}=4, r(t)=\sin (3 t)$. 


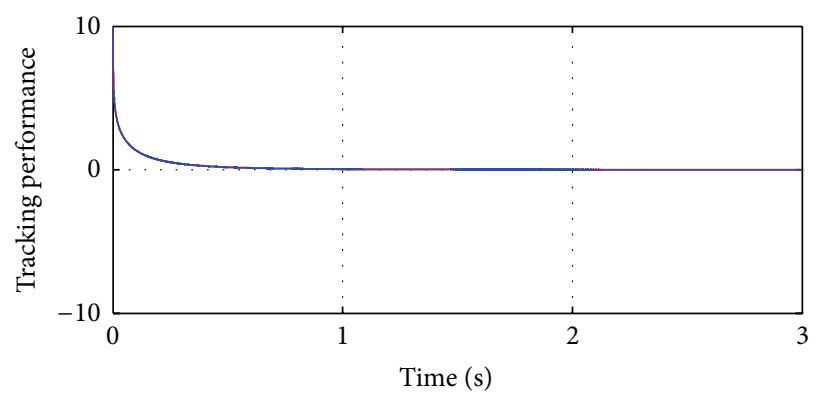

(a)

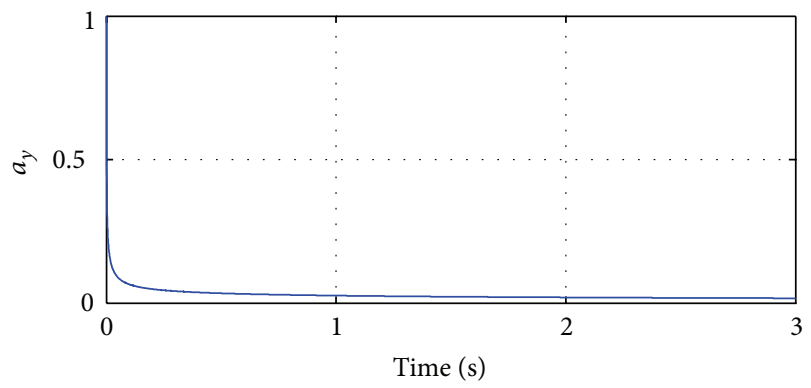

(c)

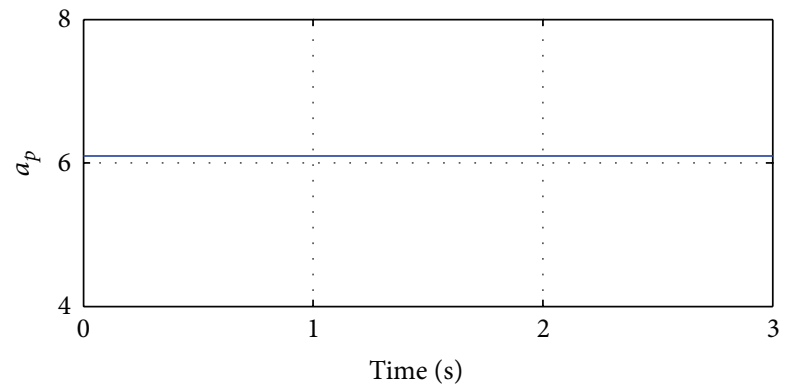

(e)

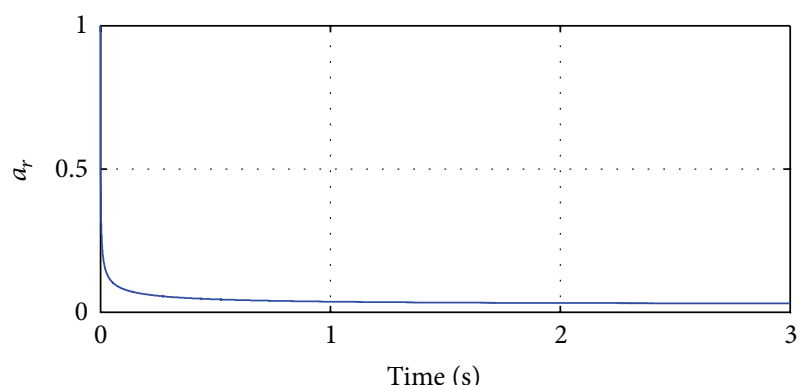

(b)

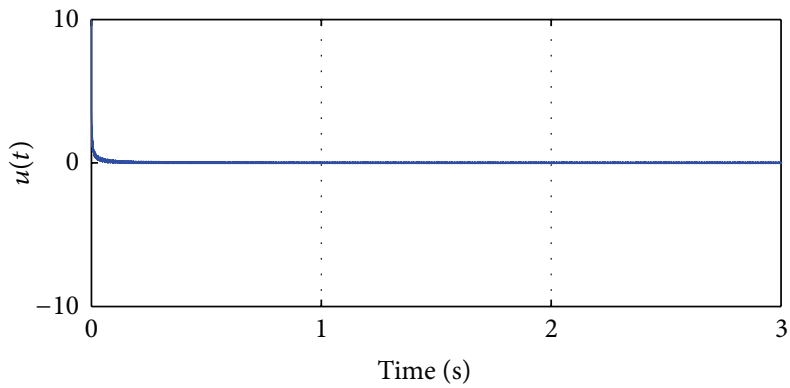

(d)

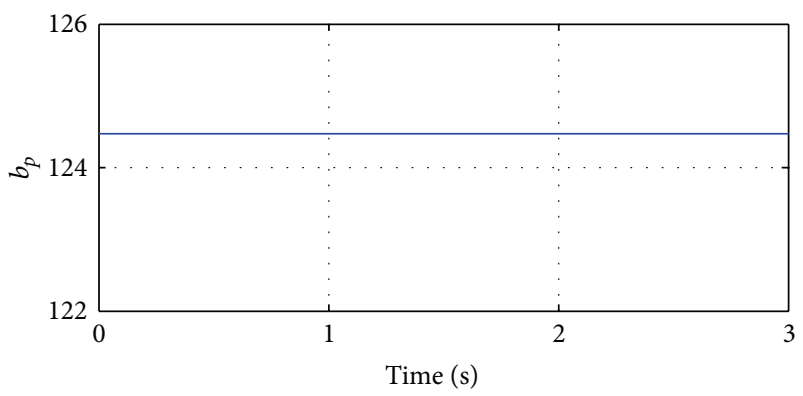

(f)

FIGURE 1: Fractional adaptive control of the fractional linear system (6). (a) Tracking performance (red line represents the state of the fractional plant, while the blue line represents the state of the fractional reference model); (b) control parameter $\widehat{a}_{r}(t) ;(\mathrm{c})$ control parameter $\widehat{a}_{y}(t) ;(\mathrm{d})$ control input $u(t)$; (e) estimation of parameter $a_{p}(t)$; (f) estimation of parameter $a_{y}(t)$.

The adaptation gain is chosen to be $\gamma=1$, while the fractional orders of the adaptation law are chosen as $\alpha_{2}=0.4$, $\alpha_{3}=0.4$.

As for the initialization issue, we refer to the method proposed by Lorenzo and Hartley in [37], where it is addressed that the initial conditions for fractional differential equations with order between 0 and 1 are a constant function of time. Therefore, the initial conditions of the fractional plant, the fractional model, and the fractional adaptation law are chosen, respectively, as $y(t)=y\left(0^{+}\right)=10, y_{m}(t)=y_{m}\left(0^{+}\right)=$ $10, \widehat{a}_{r}(t)=\widehat{a}_{r}\left(0^{+}\right)=1, \widehat{a}_{y}(t)=\widehat{a}_{y}\left(0^{+}\right)=1$, for $-\infty \leq t \leq 0$.

The numerical simulations of the behavior of the fractional linear adaptive system are illustrated in Figure 1. For interpretations of the references to the color in the upper left figure, the reader is referred to the web version of this paper.

Remark 6. In [25], the authors have designed a commensurate fractional adaptation law for the integer order SISO systems. Benefits from the use of fractional calculus are also illustrated mainly via numerical simulations. However, detailed theoretical analysis is left out in their work. In the following, we give the theoretical analysis of the fractional control for the integer order plant.

With the first order error dynamics (i.e., $\alpha_{1}=1$ in system (13)) and the fractional adaptation law (12), the closed-loop adaptive system is described by

$$
\begin{gathered}
\frac{d e}{d t}=-a_{m} e+b_{p}\left(\tilde{a}_{r} r+\tilde{a}_{y} y\right), \\
D^{\alpha_{2}} \widehat{a}_{r}=-\operatorname{sign}\left(b_{p}\right) \text { yer } \\
D^{\alpha_{3}} \widehat{a}_{y}=-\operatorname{sign}\left(b_{p}\right) \text { yey. }
\end{gathered}
$$




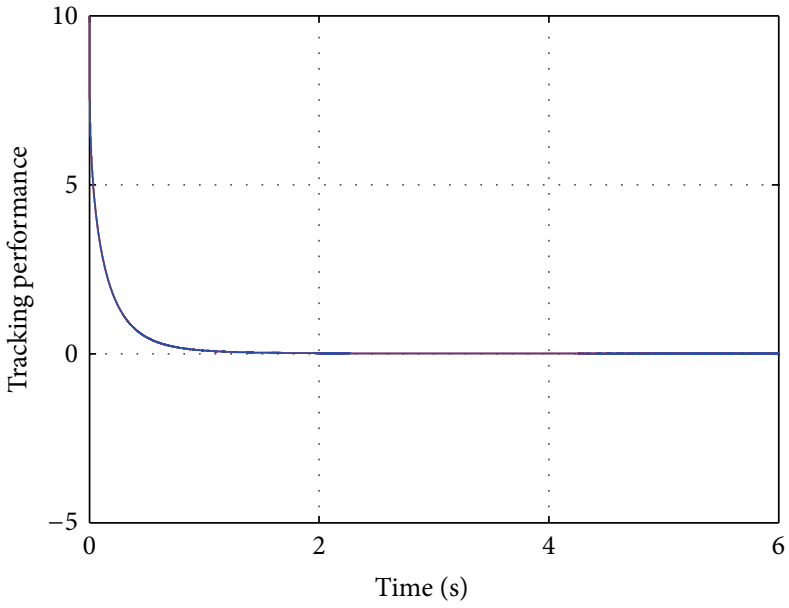

(a)

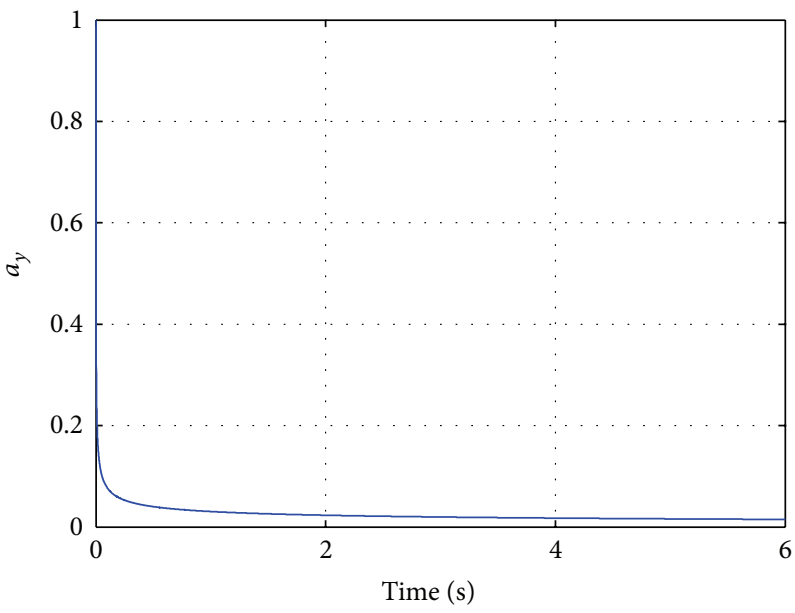

(c)

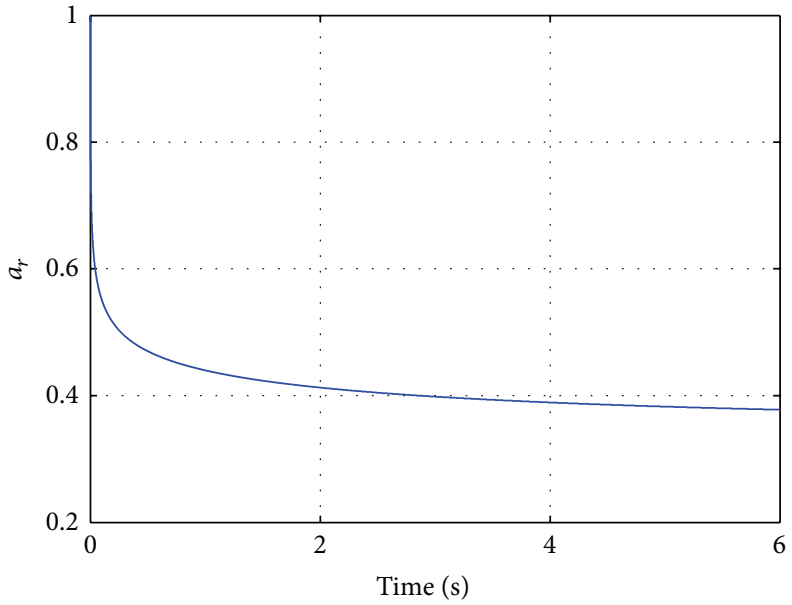

(b)

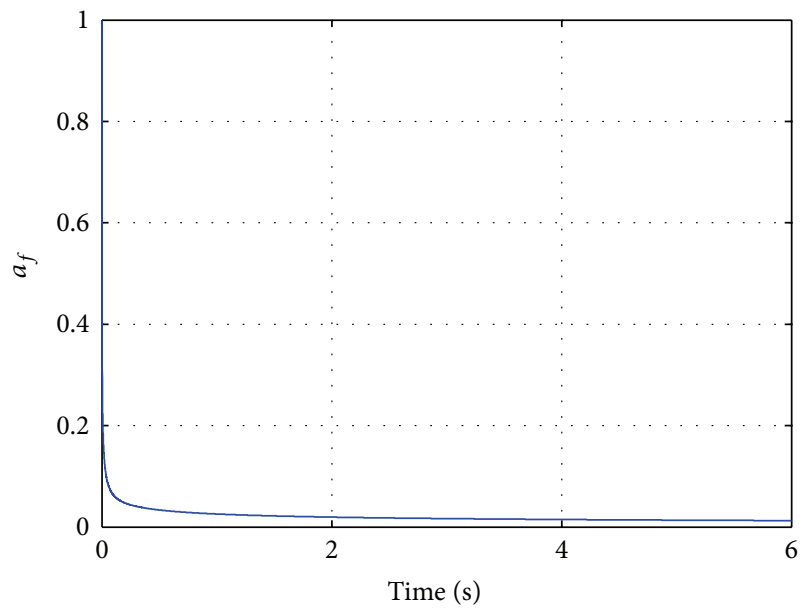

(d)

Figure 2: Fractional adaptive control of the fractional nonlinear system (26). (a) Tracking performance (red line represents the state of the fractional nonlinear plant, while, blue line represents the state of the fractional reference model); (b) control parameter $\widehat{a}_{r}(t)$; (c) control parameter $\widehat{a}_{y}(t)$; (d) control parameter $\widehat{a}_{f}(t)$.

By converting the last two FDEs into infinite-dimensional ODEs as (14) and introducing Lyapunov function as

$$
V(t)=\frac{1}{2} e^{2}+\frac{\left|b_{p}\right|}{2 \gamma} \int_{0}^{\infty} \mu_{2}(\omega) z_{2}^{2} d \omega+\frac{\left|b_{p}\right|}{2 \gamma} \int_{0}^{\infty} \mu_{3}(\omega) z_{3}^{2} d \omega
$$

one derives

$$
\begin{aligned}
\frac{d V}{d t}(t)= & -\frac{\left|b_{p}\right|}{\gamma} \int_{0}^{\infty} \mu_{2}(\omega) \omega z_{2}^{2} d \omega \\
& -\frac{\left|b_{p}\right|}{\gamma} \int_{0}^{\infty} \mu_{3}(\omega) \omega z_{3}^{2} d \omega-a_{m} e^{2} .
\end{aligned}
$$

By Lemma $5, d V / d t$ is negative semidefinite, which implies the stability of the fractional adaptive system (23). This proves that the integer order plant $\left(\alpha_{1}=1\right.$ in system (6)) can be controlled with the control law (8) and the fractional adaptation law (12).
Remark 7. From the analysis in Remark 6, it is evident that the values of the fractional orders $\alpha_{1}, \alpha_{2}$, and $\alpha_{3}$ can be extended to the following values: $0<\alpha_{1} \leq 1,0<\alpha_{2} \leq 1$, and $0<\alpha_{3} \leq 1$.

\section{Extension to Fractional Nonlinear Systems}

In this section, we extend the fractional control method previously proposed to fractional nonlinear systems. The fractional nonlinear plant is described by the fractional differential equation as follows:

$$
D^{\alpha_{1}} y=-a_{p} y-c_{p} f(y)+b_{p} u,
$$

where $f$ is a known nonlinear function. The fractional reference model is chosen as (7). Instead of using control law (8) and adaptation law (12), we now use the following control law:

$$
u=\widehat{a}_{r}(t) r+\widehat{a}_{y}(t) y+\widehat{a}_{f}(t) f(y),
$$




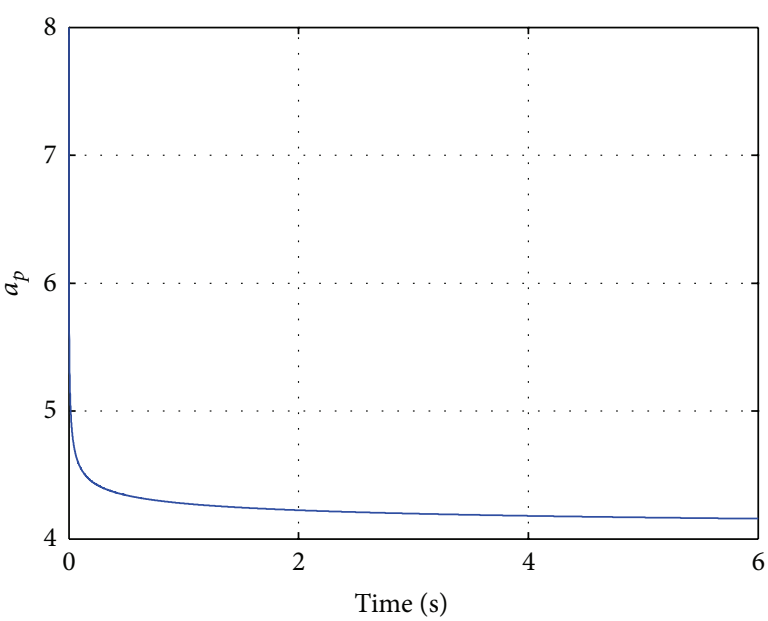

(a)

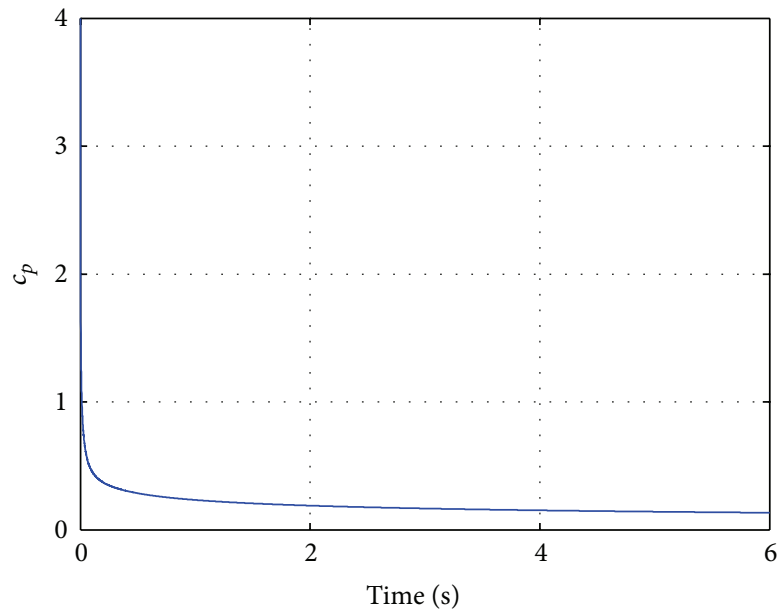

(c)

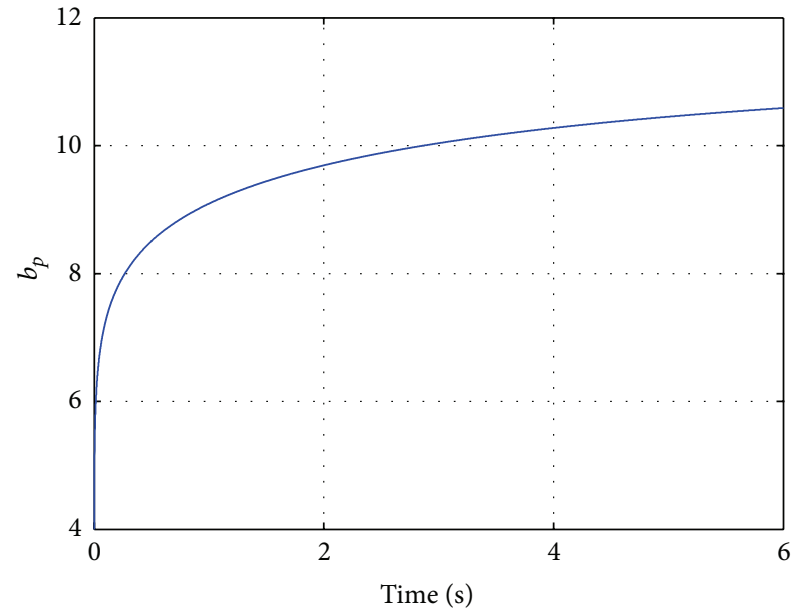

(b)

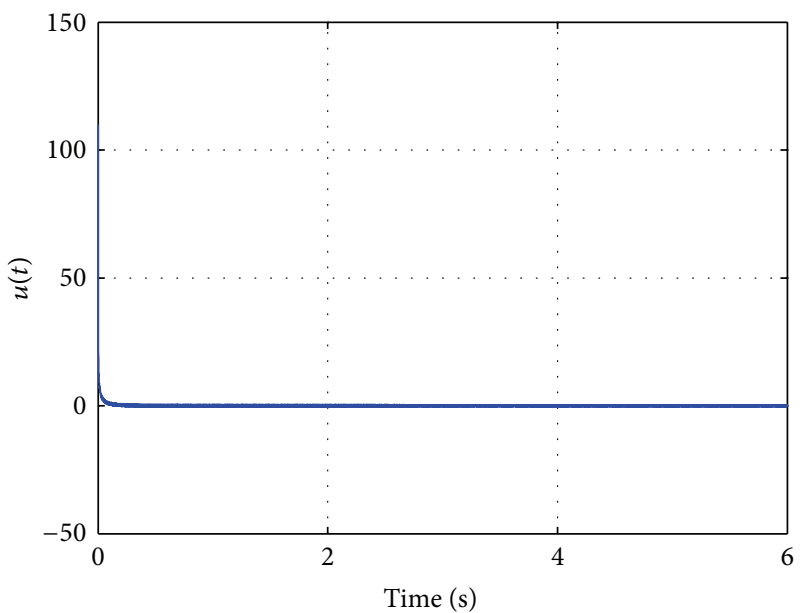

(d)

FIGURE 3: Fractional adaptive control of the fractional nonlinear system (26). (a) Estimation of parameter $a_{p}(t)$; (b) parameter $b_{p}(t)$; (c) estimation of parameter $c_{p}(t) ;(\mathrm{d})$ control input $u(t)$.

and the adaptation law

$$
\begin{aligned}
& D^{\alpha_{2}} \widetilde{a}_{r}=-\operatorname{sign}\left(b_{p}\right) \text { yer }, \\
& D^{\alpha_{3}} \tilde{a}_{y}=-\operatorname{sign}\left(b_{p}\right) \text { rey, } \\
& D^{\alpha_{4}} \widetilde{a}_{f}=-\operatorname{sign}\left(b_{p}\right) \gamma e f(y),
\end{aligned}
$$

with $\tilde{a}_{f}=\widehat{a}_{f}-c_{p} / b_{p}$.

Similarly, one can easily analyze the stability and tracking convergence of the above fractional nonlinear adaptive system based on the continuous frequency distributed model of fractional integrator.

The following example demonstrates the behavior of the fractional nonlinear adaptive system.

Consider the fractional nonlinear plant with known fractional order $\alpha_{1}=0.9$ and unknown parameters $a_{p}, b_{p}$, and $c_{p}$. The sign of $b_{p}$ is assumed to be positive. $f(y)$ is chosen to be $y^{2}$. The fractional reference model is chosen to be the same as (22).
The initial conditions of the fractional plant, the fractional model, and the fractional adaptation law are chosen, respectively, as $y(t)=y\left(0^{+}\right)=10, y_{m}(t)=y_{m}\left(0^{+}\right)=10$, $\widehat{a}_{r}(t)=\widehat{a}_{r}\left(0^{+}\right)=1, \widehat{a}_{y}(t)=\widehat{a}_{y}\left(0^{+}\right)=1, \widehat{a}_{f}(t)=\widehat{a}_{f}\left(0^{+}\right)=1$, for $-\infty \leq t \leq 0$.

The adaptation gain is chosen to be $\gamma=1$, while the fractional orders of the adaptation law are chosen as $\alpha_{2}=0.9$, $\alpha_{3}=0.6, \alpha_{4}=0.6$.

Figures 2 and 3 illustrate the numerical simulations of the behavior of the fractional nonlinear adaptive system. For interpretations of the references to the color in the upper left figure of Figure 2, the reader is referred to the web version of this paper.

\section{Concluding Remarks}

Based on the theory of fractional calculus, this paper has extended the conventional MRAC systems to fractional ones by designing a control law and a fractional adaptation law 
for the fractional plant and fractional reference model. The stability and tracking convergence have been analyzed using the frequency distributed fractional integrator model and Lyapunov theory. Moreover, numerical simulations of both linear and nonlinear systems have been performed to exhibit the viability and effectiveness of the proposed methodology.

As for the future perspectives, our research efforts will be focused on the following.

(i) How the fractional orders of the adaptation law affect the performance of the control system.

(ii) The optimal design of the fractional orders of the adaptation law.

(iii) Superiority of fractional MRAC systems compared to the conventional ones.

(iv) Design of fractional MRAC for general integer order or fractional order linear system, namely, $D^{\alpha} x=A x+$ $B u, 0<\alpha \leq 1$.

\section{Conflict of Interests}

The authors declare that there is no conflict of interests regarding the publication of this paper.

\section{References}

[1] I. Petráš, Fractional-Order Nonlinear Systems: Modeling, Analysis and Simulation, Springer, 2011.

[2] R. Caponetto, Fractional Order Systems: Modeling and Control Applications, vol. 72, World Scientific, 2010.

[3] I. Podlubny, Fractional Differential Equations: An Introduction to Fractional Derivatives, Fractional Differential Equations, to Methods of Their Solution and Some of Their Applications, vol. 198, Academic Press, 1998.

[4] J. T. Machado, V. Kiryakova, and F. Mainardi, "Recent history of fractional calculus," Communications in Nonlinear Science and Numerical Simulation, vol. 16, no. 3, pp. 1140-1153, 2011.

[5] C. P. Li and F. R. Zhang, "A survey on the stability of fractional differential equations," European Physical Journal, vol. 193, no. 1, pp. 27-47, 2011.

[6] Y. Q. Chen, I. Petráš, and D. Xue, "Fractional order controla tutorial," in Proceedings of the American Control Conference (ACC '09), pp. 1397-1411, St. Louis, Mo, USA, June 2009.

[7] B. J. Lurie, Three-Parameter Tunable Tilt-Integral-Derivative (TID) Controller, Google Patents, 1994.

[8] A. Oustaloup, X. Moreau, and M. Nouillant, "The crone suspension," Control Engineering Practice, vol. 4, no. 8, pp. 1101-1108, 1996.

[9] I. Podlubny, "Fractional-order systems and $\mathrm{PI}^{\lambda} \mathrm{D}^{\mu}$-controllers," IEEE Transactions on Automatic Control, vol. 44, no. 1, pp. 208214, 1999.

[10] H.-F. Raynaud and A. Zergaïnoh, "State-space representation for fractional order controllers," Automatica, vol. 36, no. 7, pp. 1017-1021, 2000.

[11] D. Xue and Y. Q. Chen, "A comparative introduction of four fractional order controllers," in Proceedings of the 4th World Congress on Intelligent Control and Automation, pp. 3228-3235, Shanghai, China, June 2002.
[12] J. Yuan, B. Shi, W. Ji et al., "Sliding mode control of the fractional order unified chaotic system," Abstract and Applied Analysis, vol. 2013, Article ID 397504, 13 pages, 2013.

[13] C. Yin, S.-M. Zhong, and W.-F. Chen, "Design of sliding mode controller for a class of fractional-order chaotic systems," Communications in Nonlinear Science and Numerical Simulation, vol. 17, no. 1, pp. 356-366, 2012.

[14] A. Razminia and D. Baleanu, "Complete synchronization of commensurate fractional order chaotic systems using sliding mode control," Mechatronics, vol. 23, no. 7, pp. 873-879, 2013.

[15] S. Bao, J. Yuan, and D. Chao, "Pseudo-state sliding mode control of fractional SISO nonlinear systems," Advances in Mathematical Physics, vol. 2013, Article ID 918383, 7 pages, 2013.

[16] M. P. Aghababa, "Robust stabilization and synchronization of a class of fractional-order chaotic systems via a novel fractional sliding mode controller," Communications in Nonlinear Science and Numerical Simulation, vol. 17, no. 6, pp. 2670-2681, 2012.

[17] D. M. Senejohnny and H. Delavari, "Active sliding observer scheme based fractional chaos synchronization," Communications in Nonlinear Science and Numerical Simulation, vol. 17, no. 11, pp. 4373-4383, 2012.

[18] M. P. Aghababa, "Finite-time chaos control and synchronization of fractional-order nonautonomous chaotic (hyperchaotic) systems using fractional nonsingular terminal sliding mode technique," Nonlinear Dynamics, vol. 69, no. 1-2, pp. 247-261, 2011.

[19] S. Dadras and H. R. Momeni, "Fractional terminal sliding mode control design for a class of dynamical systems with uncertainty," Journal of Science Communication, vol. 17, no. 1, pp. 367377,2012

[20] A. Si-Ammour, S. Djennoune, and M. Bettayeb, "A sliding mode control for linear fractional systems with input and state delays," Communications in Nonlinear Science and Numerical Simulation, vol. 14, no. 5, pp. 2310-2318, 2009.

[21] M. R. Faieghi, H. Delavari, and D. Baleanu, "A note on stability of sliding mode dynamics in suppression of fractional-order chaotic systems," Computers \& Mathematics with Applications, vol. 66, no. 5, pp. 832-837, 2013.

[22] M. Pourmahmood, S. Khanmohammadi, and G. Alizadeh, "Synchronization of two different uncertain chaotic systems with unknown parameters using a robust adaptive sliding mode controller," Communications in Nonlinear Science and Numerical Simulation, vol. 16, no. 7, pp. 2853-2868, 2011.

[23] R. Zhang and S. Yang, "Robust synchronization of two different fractional-order chaotic systems with unknown parameters using adaptive sliding mode approach," Nonlinear Dynamics, vol. 71, no. 1-2, pp. 269-278, 2012.

[24] Y. Jian, S. Bao, Z. Xiaoyun et al., "Sliding mode control of the fractional order unified chaotic system," Abstract and Applied Analysis, vol. 2013, Article ID 397504, 13 pages, 2013.

[25] B. M. Vinagre, I. Petráš, I. Podlubny, and Y. Q. Chen, "Using fractional order adjustment rules and fractional order reference models in model-reference adaptive control," Nonlinear Dynamics, vol. 29, no. 1-4, pp. 269-279, 2002.

[26] S. Ladaci and A. Charef, "On fractional adaptive control," Nonlinear Dynamics, vol. 43, no. 4, pp. 365-378, 2006.

[27] Z. M. Odibat, "Adaptive feedback control and synchronization of non-identical chaotic fractional order systems," Nonlinear Dynamics, vol. 60, no. 4, pp. 479-487, 2010.

[28] C. Li and Y. Tong, "Adaptive control and synchronization of a fractionalorder chaotic system," Pramana, vol. 80, no. 4, pp. 583-592, 2013. 
[29] L. Chen, S. Wei, Y. Chai, and R. Wu, "Adaptive projective synchronization between two different fractional-order chaotic systems with fully unknown parameters," Mathematical Problems in Engineering, vol. 2012, Article ID 916140, 16 pages, 2012.

[30] O. P. Agrawal, "A general formulation and solution scheme for fractional optimal control problems," Nonlinear Dynamics, vol. 38, no. 1-4, pp. 323-337, 2004.

[31] Z. D. Jelicic and N. Petrovacki, "Optimality conditions and a solution scheme for fractional optimal control problems," Structural and Multidisciplinary Optimization, vol. 38, no. 6, pp. 571581, 2009.

[32] S. Djennoune and M. Bettayeb, "Optimal synergetic control for fractional-order systems," Automatica, vol. 49, no. 7, pp. 22432249, 2013.

[33] J. C. Trigeassou, N. Maamri, J. Sabatier, and A. Oustaloup, "A Lyapunov approach to the stability of fractional differential equations," Signal Processing, vol. 91, no. 3, pp. 437-445, 2011.

[34] J. Yuan, S. Bao, and J. Wenqiang, "Adaptive sliding mode control of a novel class of fractional chaotic systems," Advances in Mathematical Physics, vol. 2013, Article ID 576709, 13 pages, 2013.

[35] J. J. E. Slotine and W. Li, Applied Nonlinear Control, vol. 1, Prentice Hall, Upper Saddle River, NJ, USA, 1991.

[36] K. J. Åström and B. Wittenmark, Adaptive Control, Courier Dover, 2008.

[37] J. Sabatier, O. P. Agrawal, and J. Machado, Advances in Fractional Calculus: Theoretical Developments and Applications in Physics and Engineering, Springer, 2007. 


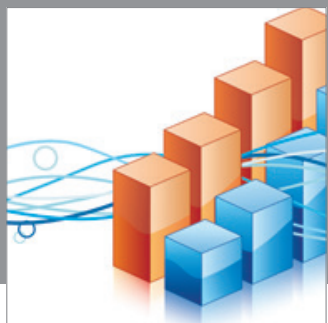

Advances in

Operations Research

mansans

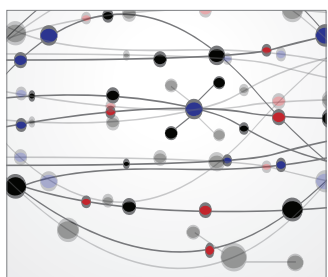

The Scientific World Journal
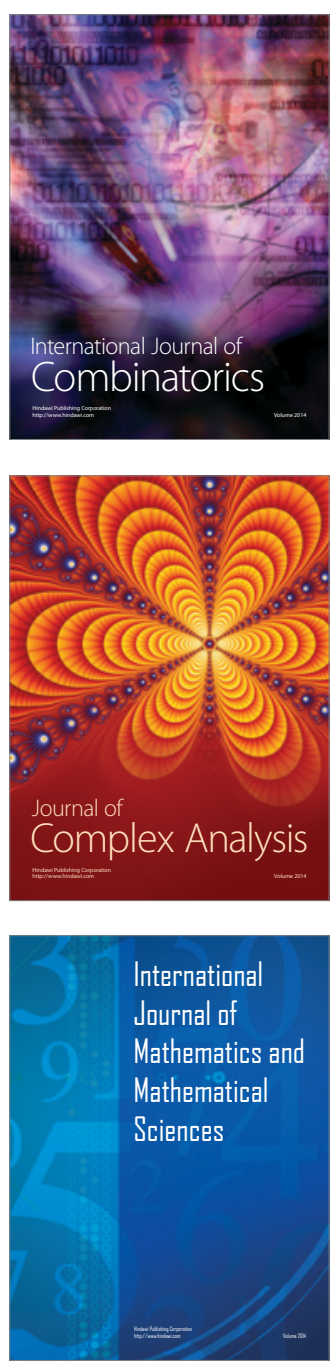
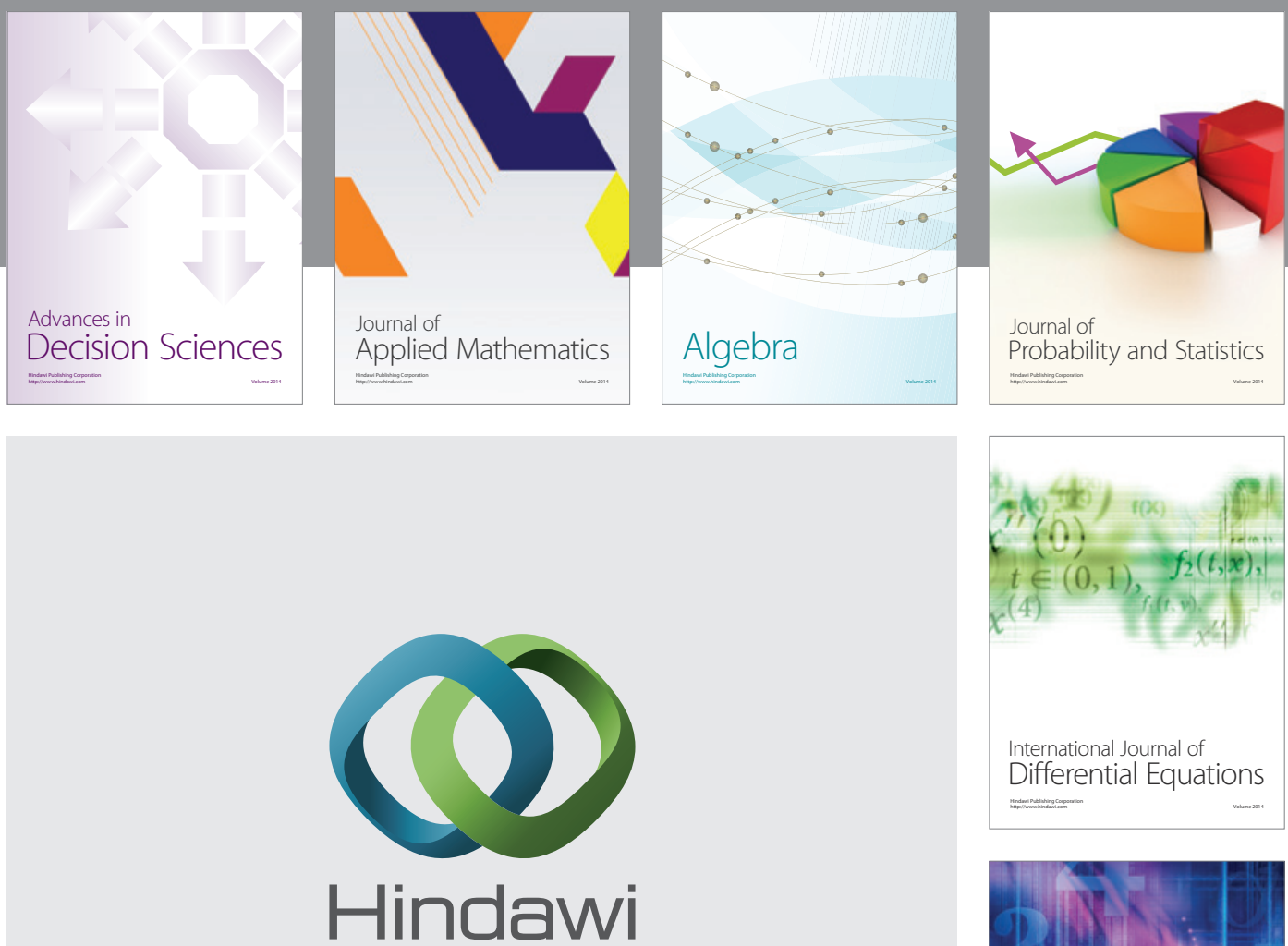

Submit your manuscripts at http://www.hindawi.com
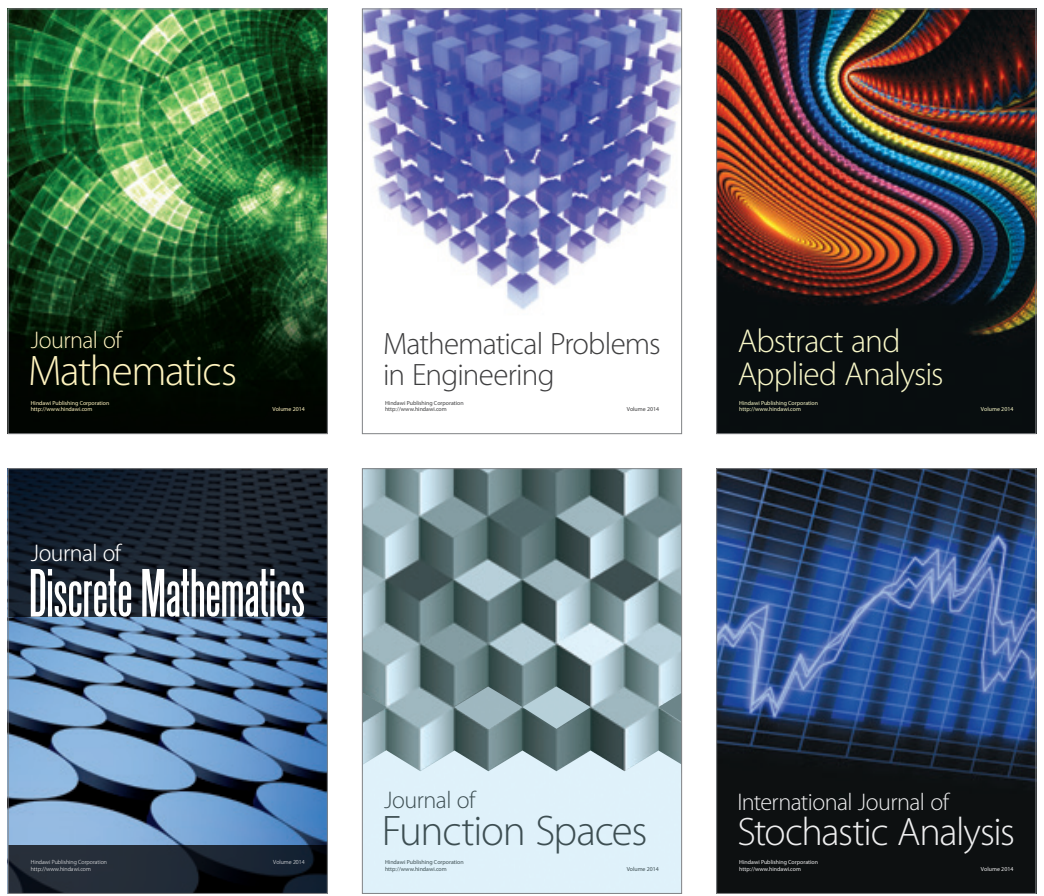

Journal of

Function Spaces

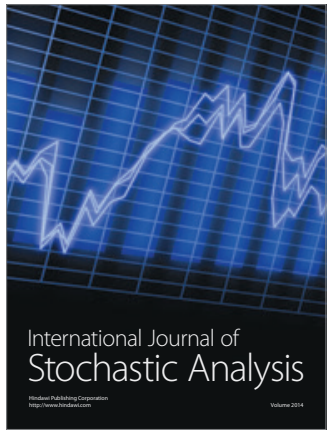

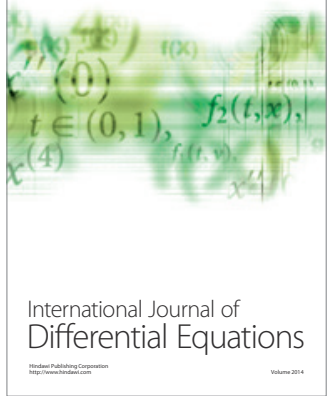
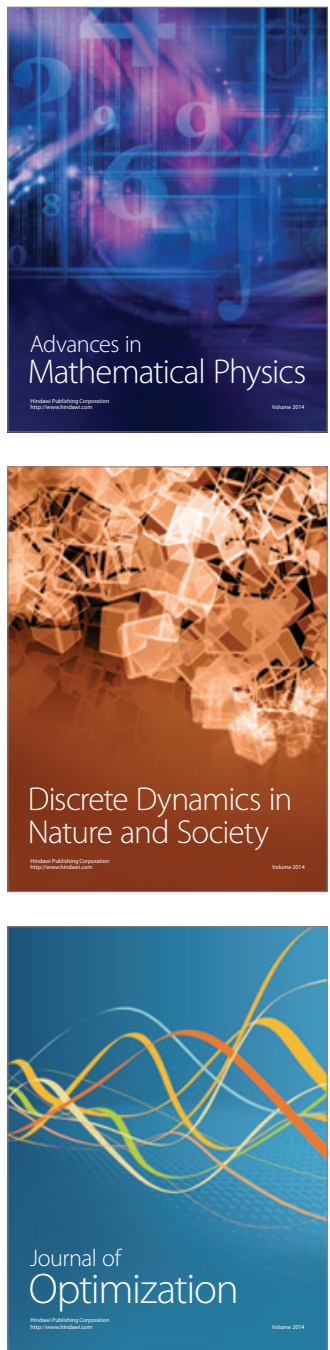